\title{
Formulation and Optimization of Pluronic Lecithin Organogel Containing Verapamil Hydrochloride Using Factorial Design Method
}

\author{
Sandeep C Atram ${ }^{\text {a* }}$ and Shrikant D. Pande ${ }^{a}$ \\ aVidyabharati College of Pharmacy, Amravati, Maharashtra, India - 444602.
}

Received: 24 Oct 2020 / Accepted: 22 Nov 2020/ Published online: 01 Jan 2021

*Corresponding Author Email: sandyphp@gmail.com

\begin{abstract}
The purpose of the current study was to investigate the feasibility of pluronic lecithin organogel as transdermal drug delivery system of verapamil hydrochloride. Verapamil hydrochloride is an L-type calcium channel blocker of the phenylalkylamine class. Formulations were developed using $30 \%$ oil phase and $70 \%$ aqueous phase. A $3^{2}$ factorial design was applied to check the effect of independent variables, Pluronic $F-127\left(X_{1}\right)$ and Soya-Lecithin $\left(X_{2}\right)$ on the dependent variable $(Y)$ Percentage drug release in $24 \mathrm{hr}$. The formulated organogels were evaluated for physicochemical properties, and in vitro diffusion study. It was found that the $\mathrm{pH}$ of all the formulations is in the range of to 6-7 that suits the skin $\mathrm{pH}$, indicating skin compatibility. Invitro permeation study were performed using modified KC diffusion cell. Kinetic data revealed that the drug release followed Korsemeyer-Peppas model and the mechanism of release was found to be non fickian diffusion. The search for the optimized formulation composition was carried out using the desirability function approach with Design Expert $^{\mathrm{TM}}$ 11.1.2.0, criterion being one having the maximum desirability value. The suggested formulation was considered as optimized batch (VOT3). Hence, Pluronic lecithin organogel is efficient carrier for transdermal drug delivery of verapamil hydrochloride, thereby sustaining the action.
\end{abstract}

\section{Keywords}

Pluronic Lecithin organogel, Verapamil hydrochloride, Transdermal drug delivery system, Factorial design, Optimization.

\section{INTRODUCTION:}

Transdermal drug delivery systems are selfcontained discrete dosage forms that, when applied to the intact skin, deliver the drug through the skin at a controlled rate to the systemic circulation. Transdermal delivery has many advantages over conventional modes of drug administration, it avoids hepatic first pass metabolism and improves patient compliance. [1-2] Organogels are bicontineous colloidal system that coexist as micro heterogeneous solid (i.e. Gelator) and organic liquid phase. In general, organogels formation is based in the spontaneous self-assembly of individual gelator molecules into three-dimensional networks of randomly entangled fiber like structures. This three dimensional network holds micro domains of the 
liquid in a non-flowing state mainly through surface tension. Lecithin Organogels, the jelly like phases, consist of a 3-dimensional network of entangled reverse cylindrical (polymer-like) micelles, which immobilizes the continuous or macroscopic external organic phase, thus turning a liquid into a gel. ${ }^{[3-7]}$ The formation of transition at the micellar level in a low viscous Newtonian liquid, consisting of lecithin reverse micelles in non-polar organic liquid. However, these systems can also be called as polymer like micelles or wormlike or threadlike micelles. ${ }^{[7,8]}$

Verapamil hydrochloride is an L-type calcium channel blocker of the phenylalkylamine class. Given orally, 90 to $100 \%$ of verapamil is absorbed, but due to high first-pass metabolism, bioavailability is much poorer (10 to35\%). It is $90 \%$ bound to plasma proteins and it has a volume of distribution of 3 to $51 / \mathrm{kg}$. It gets metabolized in the liver into 12 inactive metabolites. $70 \%$ of metabolites get excreted in the urine and $16 \%$ in feces; 3 to $4 \%$ is excreted unaffected in urine. Metabolism is a nonlinear dependence between plasma concentration and dosage. Onset of action is 1 to 2 hours after oral dosage. $t 1 / 2$ is 2.8 to 7.4 hours. ${ }^{[9,10]}$

Skin is a natural barrier against the penetration of most drugs, and the ability of drug to penetrate the skin epidermis, dermis, and subcutaneous fat layers depend on the properties of the drug and the carrier base. Traditional topical drug delivery systems like emulsion and suspension have various limitations such as instability and minimal systemic absorption of drug. Therefore the objective of the present study is development of a novel topical vehicle for the delivery of an antihypertensive agent in terms of effectiveness and elegance as compared to conventional drug delivery system, improved availability at the desired site by use of organogels and improved patient compliance.

\section{MATERIALS AND METHOD: \\ Material}

Verapamil hydrochloride was procured from Yarrow Chemicals, Mumbai, India. Poloxamer (Pluronic F127) were provided a gift samples by M/s BASF corporation, NJ, USA. Soya Lecithin and sorbic acid were procured from $\mathrm{M} / \mathrm{s}$ Himedia labs Pvt. Ltd. Mumbai, India. Isopropyl myristate was a product of Central Drug House Pvt. Ltd, New Delhi, India. All other chemicals used were of analytical grade.

Methods [2, 11-14]

The various formulations of Pluronic Lecithin Organogel (Table1) were developed with different compositions. The oil phase was prepared by dispersing the specific amount of soya lecithin at room temperature in isopropyl myristate (IPM). The mixture was kept overnight at room temperature to dissolve its constituents. Then sorbic acid (SA) in appropriate quantity was added, finally, drug and plasticizer were dissolved in an oil phase. The aqueous phase was prepared by dispersing the weighed amount of Pluronic F-127 and potassium sorbate (PS) in cold water. The dispersion was stored in a refrigerator overnight for effective dissolution of Pluronic F-127. Finally, aqueous phase (70\%) was slowly added in oil phase (30\%) with stirring at 400 rpm using mechanical stirrer. The formulation shown in table no.1.

\section{Experimental design}

The formulation compositions are optimized by factorial design following $3^{2}$ factorial analysis design. The formulae were developed as 9 sets varying the variables (polymer combination and Penetration enhancer) following $3^{2}$ full factorial design (3 levels) using Design Expert ${ }^{\mathrm{TM}}$ 11.1.2.0. The two independent variables selected were the concentration of pluronic $\mathrm{F}-127\left(\mathrm{X}_{1}\right)$ and Soya-Lecithin $\left(\mathrm{X}_{2}\right)$. The dependent variable selected for the response was drug release in $24 \mathrm{hr}$. The concentration range of independent variables was selected based on the following observations from preliminary experimentation. The selected concentration range of independent variables under study with their low, medium and high levels is shown in Table 1.

\section{Organogel Characterization}

Organoleptic characteristics ${ }^{[15]}$

Each formulation was tested for color, odor, texture and phase separation as well as feels upon application (stiffness, grittiness, greasiness and tackiness).

\section{Homogeneity test}

A small quantity of each gel was pressed between the thumb and the index finger in order to notice the consistency of cream that any coarse particles being attached or detached on the finger.

\section{Conclusiveness}

Prepared gel was taken in vial and visually observed weather it is occlusive or not.

\section{pH Determination}

The $\mathrm{pH}$ of formulated organogels was determined using $\mathrm{pH}$ meter. A solution containing $1 \mathrm{gm}$ of organogel in $30 \mathrm{ml}$ of neutralized distilled water was prepared and subjected to $\mathrm{pH}$ measurement. The results are given in the table-4.

\section{Rheological Measurement}

The rheological properties of organogel were measured using a thermostatically controlled Brookfield programmable rheometer (Model DV-1+ Brookfield viscometer) by using concentric cylinder 
spindle LV-4 at $100 \mathrm{rpm}$ and at temperature $25 \circ \mathrm{C}$. The results are given in the table- 4 .

Spreadability ${ }^{[16]}$

Spreadability of formulations was determined by an apparatus suggested by Multimer et al. which was fabricated in laboratory and used for study. The apparatus consists of a wooden block, with a fixed glass slide and movable glass slide with one end tied to weight pan rolled on the pulley, which was in horizontal level with fixed slide. An excess of gel sample $2.5 \mathrm{~g}$ was placed between two glass slides and a $1000 \mathrm{~g}$ weight was placed on slides for 5 minutes to compress the sample to a uniform thickness. The time (seconds) required to separate the two slides was taken as a measure of Spreadability. It was calculated using the formula, $\mathbf{S}=\mathbf{M L} / \mathbf{T}$

Where, $\mathrm{S}$ is spreadability in $\mathrm{gcm} / \mathrm{sec}, \mathrm{M}$ is weight tied to upper slide, $L$ is length of glass slide, $T$ is time in seconds. The results are given in the table- 4 .

Drug Content ${ }^{[17]}$

The content of verapamil hydrochloride in the formulations was determined as per the method described by Willaimann et.al. For estimation verapamil hydrochloride content $1 \mathrm{gm}$ of organogel was diluted to $100 \mathrm{ml}$ with phosphate buffer $\mathrm{pH} 7.4$ and analysed spectrophotometrically at $278 \mathrm{~nm}$. The results are given in the table-4.

In vitro Drug Permeation Studies ${ }^{[18]}$

In-vitro diffusion study of formulated organogel was carried out on Franz diffusion cell having $2.0 \mathrm{~cm}$ diameter and $18 \mathrm{ml}$ capacity. Dialysis membrane (Himedia) having a molecular weight cut-off range 12000-14000kDa was used as a diffusion membrane. The diffusion cell was filled with phosphate buffer $\mathrm{pH}$ 7.4; dialysis membrane was mounted on the cell. The temperature was maintained at $34 \pm 0.5 \circ \mathrm{C}$. At predetermined time points, $1 \mathrm{ml}$ samples were withdrawn from the acceptor compartment, replacing the sampled volume with phosphate buffer pH 7.4 after each sampling, for 540minutes. The samples withdrawn were filtered and used for analysis. The amount of permeated drug was determined using a UV- spectrophotometer at $278 \mathrm{~nm}$. The results are given in the table- 4 .

\section{Kinetics of the drug release:}

To know the mechanism of the drug release from the patch's, the results obtained from the in-vitro permeation studies were analysed by various kinetic models. 1. Zero order drug release: cumulative \% drug release Vs time. 2. First order drug release: log cumulative\% drug retained Vs time 3. Higuchi's diffusion equation: cumulative \%drug release Vs square root of time 4. Peppas-korsemeyer exponential: log cumulative \% drug release Vs log time. The results are given in the table- 5 .

Stability Studies:

Stability of a drug has been defined as the ability of a particular formulation, in a specific container, to remain within its physical, chemical, therapeutic and toxicological specifications. In the present study, stability studies were carried out at $2-8^{\circ} \mathrm{C}$, room temperature and at $40 \circ \mathrm{\circ} \pm 2{ }^{\circ} \mathrm{C} / 75 \% \mathrm{RH} \pm 5 \%$ for a specific time period up to 3 months for the optimized formulation. The evaluation parameters check periodically. The results are given in the table-6.

\section{RESULT AND DISCUSSION:}

\section{Formulation and Characterization of Pluronic} Organogel Formulations

In the present work, pluronic lecithin organogel was prepared for transdermal delivery of verapamil hydrochloride with improved therapeutic performance by their penetration enhancing property. In order to achieve this objective, the formulations were prepared with soya lecithin, isopropyl myristate and polyethylene glycol 600 as a co surfactant. The formulations were characterized for their organoleptic properties, homogeneity, conclusiveness, $\mathrm{pH}$, viscosity.

The physiological observation shows that organogel formulations were washable, yellowish in color with no phase separation, smooth in feel and showed no clogging which indicate good texture of system. Freedom from grittiness reflects the degree of acceptability of verapamil hydrochloride formulation by the patients.

pH:

The $\mathrm{pH}$ of the organogels was measured at room temperature by using an electrode-based digital $\mathrm{pH}$ meter. The $\mathrm{pH}$ of all formulations ranged between $5.30 \pm 0.15-6.30 \pm 0.15$ indicating their probable use in the formulation of the transdermal or topical products.

\section{Viscosity:}

The viscosity values were measured for organogel formulations using Brookfield viscometer DV-E with spindle no. 7 at $50 \mathrm{rpm}$. The viscosity ranged from 96500-188000cp.

\section{Spreadability:}

The spreadable nature of organogel was checked. Spreadability of all the batches shows in the range $12.79-25.11 \%$, results for the spreading ability of organogel.

\section{Drug Content:}

The content of organogel formulations was found in the range of $97.58 \pm 2.10-100.34 \pm 0.22$ indicating uniform distribution of drug through the base and no interaction of drug with component of base. 
Invitro Permeation Studies:

Invitro permeation studies were carried out for all 9 formulations using dialysis membrane as barrier in phosphate buffer $\mathrm{pH}$ 7.4. The maximum and minimum drug release obtained for optimized are 86.25 and 92.15, respectively. The verapamil hydrochloride release was found to be decreased with an increase in soyabean oil content, an inverse correlation exist between the release rate and the gel viscosity value. But a significant decrease in verapamil hydrochloride release was obtained as the soyabean oil concentration was further increase. Further increase in concentration of organogelator decrease cumulative percentage drug release which might be due to extensive entanglement of long cylindrical micelles with each other, forming network like structure with increase in viscosity.

\section{Kinetics of Drug Release data:}

In this study, different formulations released variable amount of verapamil hydrochloride through membrane into the invitro fluid. To study the drug diffusion kinetics and mechanism the results were fitted to zero, first order, Higuchi and the release data were also analyzed as per Korsemeyer-Peppa's plot.

The invitro permeation data were fit to different equations and kinetic models to explain permeation profile (Table No. 5, Fig No. 1). The coefficient of correlation of each of the kinetics was calculated and compared. The invitro permeation profiles of all the different formulations of $\mathrm{VH}$ organogel did not fit to zero order behaviour truly and they could be beat expressed by Higuchi's equation for the release of drug from organogel system that depends on rate controlling parameters in the drug release. The data was further treated as per Korsemeyer-Peppas equation. It was observed that the release exponent $\mathrm{n}$ was 0.5 , this suggested that an anomalous or non fickian diffusion. Batch VOT 3 has $R^{2}$ value $(0.9823)$ and $n$ value $(0.6572)$. The slope $(n)$ values obtained by this equations indicated that the drug released by Fickian diffusion predominated with all formulations. EXPERIMENTAL DESIGN ${ }^{[19-21]}$

The evaluation of data for optimization process was carried out by Design Expert Software ${ }^{\mathrm{TM}}$ 11.1.2.0. The $3^{2}$ factorial design was used to optimize the formulation variables and the data generated was used to fit in a quadratic polynomial equations for dependent variable as shown in the equation 1.

$y=\beta_{0}+\beta_{1} X_{1}+\beta_{2} X_{2}+\beta_{3} X_{1} X_{2}+\beta_{4} X_{1}{ }^{2}+\beta_{5} X_{2}{ }^{2}+\varepsilon \ldots \ldots \ldots .1$ In this equation, $y$ is a dependent variable; $\beta_{1}, \beta_{2}, \beta_{3}$, $\beta_{4}$, and $\beta_{5}$ are constant regression coefficient of the factor (linear terms), their interaction and quadratic terms, respectively; $\beta_{0}$ is the arithmetic mean response of the 9 runs and $\varepsilon$ is random error.
For estimation of quantitative effects of the different combination of factors and the factor levels on the percentage drug release, the response models were calculated with Design Expert software by applying coded values of factor levels. The model described could be represented as:

Coded level: Percentage Drug Release $\left(\mathbf{Y}_{1}\right)=5965$ $3.03 A+2.71 B-3.20 A B-0.7950 A^{2}+3.45 B^{2} \ldots \ldots \ldots . .2$ Fitting of Data to the Model

A $3^{2}$ statistical experimental design as the RSM requires 9 experiments. Formulation 9 showed a significantly better percentage drug release among the experimental runs. All the responses observed for 9 formulations prepared were simultaneously fit to first order, second order, and quadratic models using Design Expert 11.1.2.0. It was observed that the best fit model was quadratic model and Sum of squares is Type III -partial. A positive value represents an effect that favours the optimization, while a negative value indicates an inverse relationship between the factor and response. It is evident that all the three independent variables, viz. the Pluronic F-127 $\left(X_{1}\right)$, and Lecithin $\left(X_{2}\right)$ have positive effects on the response, viz. percentage drug release.

The quantitative effects of the different combination of factors and factor levels on the percentage drug release was calculated using response surface models. The significant $p$ value $(p<0.05), R^{2}$, adjusted $R^{2}$, and coefficient of variation values of this model indicated that the assumed regression model was significant and valid for each considered response. The values of the coefficients in the model are related to the effect of these variables on the response. Form this model quadratic was best, indicating that combination of above system had the greatest potential influence on the telmisartan organogel.

$3 \mathrm{D}$ response surface plots give a representation of the variations in each response when the two factors are simultaneously changed from lower to higher level. It gives a three dimensional curvature of the change in response at different factor levels. It also gives the variation in design points from the predicted response value. The 3-D response surface (Fig. No. 2) and counter plot (Fig. No. 2) were drawn to estimate the effects of the independent variables on response and to select the optimal formulation.

\section{Data Analysis}

The percentage drug release (dependent variable) obtained at various levels of the three independent variables $\left(X_{1}\right.$, and $\left.X_{2}\right)$ was subjected to multiple regression to yield a polynomial equation. The value of correlation coefficient $\left(r^{2}\right)$ of the equation was found to be 0.9683 , indicating good fit. VIF value 
found to be 1 , indicating model is significant. According to table 6 , the result calculated using equation 2 was statistically significant with $p<0.005$, indicating that the developed model exhibited good agreement between the response $Y_{1}$ and the significant variables. The value of lack of fit for the equation more than 0.05 indicating that the proposed statistical model fit well. The ANOVA result for $Y_{1}$ provides $F$ value 18.33 as compared to the critical values from the cut off point for $F$ distribution (=0.05 (Table 145). These F value suggested that the derived quadratic models has significant influence on the response $R^{2}$ and adjusted $R^{2}$ value for $Y_{1}$ were $0.9155,0.6831$, respectively demonstrate the accuracy of the test and the fitness of the results with prepared model. The percentage drug release measured for the different formulations showed wide variation (i.e. vales ranged from 88.75 to $92.15 \%)$. The results clearly indicate that the percentage drug release is strongly affected by the variables selected for the study. The main effects of $X_{1}$, and $X_{2}$ represents the average result of changing one variable at a time from its low level to its high level. The interaction terms $\left(X_{1} X_{2}, X_{1} X_{3}, X_{2} X_{3}, X_{1}{ }^{2}, X_{2}{ }^{2}\right.$, $X_{3}{ }^{2}$ ) shows how the percentage drug release changes when two variables are simultaneously changed. The negative coefficient for all three independent variables an unfavourable effect on the percentage drug release, while positive coefficients for the interactions between two variables indicate a favourable effect on percentage drug release.

Search for the Optimum Formulation:

The search for the optimized formulation composition was carried out using the desirability function approach with Design Expert software, criterion being one having the maximum desirability value. The optimization process was performed by setting the responses within selected ranges. Target ranges for the \% drug release on TM organogel $(\mathrm{Y})$. The suggested formulation was considered as optimized batch (VOT3). The composition of VOT3 and the responses predicted by software are listed in table no. 7. Batch VOT3was found to exhibit the value of observed parameters close to the values predicted by software.

Stability Studies:

The optimized formulations was subjected to stability studies at three different temperatures (2$8 \circ \mathrm{C}, \mathrm{RT}$ and $40 \circ \mathrm{C}$ ). It was found that more than $98 \%$ of drug was present in all the formulations after 90 days at RT whereas at $2-8$ 으, there was about $2 \%$ and at $40 \circ \mathrm{C}$ there was also $2 \%$ decline in the drug content observed in the tested formulations. Other characteristics like transparency, feel and $\mathrm{pH}$ were also observed as given in the table no. 8 and no significant change was found in these characteristics, which showed that during stability studies, the prepared formulation was found to be stable without any significant changes at RT.

Table No. 1: Formulation and design of $3^{2}$ full factorial experiment design layout for Verapamil hydrochloride organogel

\begin{tabular}{lll}
\hline \multirow{2}{*}{ Batch } & \multicolumn{2}{l}{ Variable level coded formulation } \\
\cline { 2 - 3 } & $\mathbf{X}_{\mathbf{1}}$ & $\mathbf{X}_{\mathbf{2}}$ \\
\hline VOT 1 & -1 & -1 \\
VOT 2 & -1 & 0 \\
VOT 3 & -1 & +1 \\
VOT 4 & 0 & -1 \\
VOT 5 & 0 & 0 \\
VOT 6 & 0 & +1 \\
VOT 7 & +1 & -1 \\
VOT 8 & +1 & 0 \\
VOT 9 & +1 & +1 \\
\hline
\end{tabular}

Table No 2: Values of variables in $3^{2}$ full factorial design for Verapamil hydrochloride Organogel

\begin{tabular}{lll}
\hline Coded values & Actual values $\left(\mathbf{X}_{\mathbf{1}}=\right.$ Pluronic $\left.\mathbf{F}-127\right)$ & Actual values $\left(\mathbf{X}_{\mathbf{2}}=\right.$ Soya Lecithin $)$ \\
\hline-1 & 10 & 2.5 \\
0 & 15 & 5 \\
+1 & 20 & 10 \\
\hline
\end{tabular}


Table No. 3: Actual formulation design layout for Verapamil hydrochloride Organogel

\begin{tabular}{llllllllll}
\hline Content & TOT1 & TOT 2 & TOT 3 & TOT 4 & TOT 5 & TOT 6 & TOT 7 & TOT 8 & TOT 9 \\
\hline Verapamil hydrochloride (\%) & 1 & 1 & 1 & 1 & 1 & 1 & 1 & 1 & 1 \\
Lecithin Soya (\%) & 2.5 & 2.5 & 2.5 & 5 & 5 & 5 & 10 & 10 & 10 \\
Sorbic Acid (\%) & 0.2 & 0.2 & 0.2 & 0.2 & 0.2 & 0.2 & 0.2 & 0.2 & 0.2 \\
Polyethylene Glycol 600 (\%) & 10 & 10 & 10 & 10 & 10 & 10 & 10 & 10 & 10 \\
Isopropyl Myristate upto (\%) & 100 & 100 & 100 & 100 & 100 & 100 & 100 & 100 & 100 \\
Pluronic F-127 (\%) & 10 & 15 & 20 & 10 & 15 & 20 & 10 & 15 & 20 \\
Potassium Sorbate (\%) & 0.2 & 0.2 & 0.2 & 0.2 & 0.2 & 0.2 & 0.2 & 0.2 & 0.2 \\
Purified water upto (\%) & 100 & 100 & 100 & 100 & 100 & 100 & 100 & 100 & 100 \\
\hline
\end{tabular}

Table no. 4: Characterization of Verapamil hydrochloride Organogels Formulation

\begin{tabular}{llllllll}
\hline Batch & Appearance & pH & $\begin{array}{l}\text { Drug } \\
\text { Content }\end{array}$ & $\begin{array}{l}\text { Phase } \\
\text { Separation }\end{array}$ & Spreadability & $\begin{array}{l}\text { Viscosity } \\
\text { (cp) }\end{array}$ & $\begin{array}{l}\text { \% Drug } \\
\text { diffused }\end{array}$ \\
\hline VOT 1 & Good & $6.48 \pm 0.15$ & 95.132 & No & 8958 & 18.21 & 90.02 \\
VOT 2 & Good & $6.96 \pm 0.15$ & 97.416 & No & 9253 & 18.11 & 90.04 \\
VOT 3 & Good & $7.10 \pm 0.15$ & 98.768 & No & 8751 & 19.50 & 92.15 \\
VOT 4 & Good & $6.87 \pm 0.15$ & 94.960 & No & 9026 & 17.92 & 90.11 \\
VOT 5 & Good & $6.95 \pm 0.15$ & 94.960 & No & 9172 & 17.70 & 90.71 \\
VOT 6 & Good & $7.10 \pm 0.15$ & 90.192 & No & 7741 & 18.06 & 91.02 \\
VOT 7 & Good & $6.77 \pm 0.15$ & 99.392 & No & 7652 & 18.11 & 89.42 \\
VOT 8 & Good & $6.52 \pm 0.15$ & 99.564 & No & 7800 & 18.66 & 86.25 \\
VOT 9 & Good & $7.21 \pm 0.15$ & 94.192 & No & 7720 & 17.97 & 88.75 \\
\hline
\end{tabular}

Table No 5: Kinetics of drug release of formulation of Verapamil hydrochloride organogel

\begin{tabular}{llllll}
\hline \multirow{2}{*}{ Batch } & Zero order & First order & Higuchi Model & \multicolumn{2}{l}{ Korsemeyer-Peppas Model } \\
\cline { 2 - 6 } & $\mathbf{R}^{\mathbf{2}}$ & $\mathbf{R}^{\mathbf{2}}$ & $\mathbf{R}^{\mathbf{2}}$ & $\mathbf{R}^{\mathbf{2}}$ & $\mathbf{n}$ \\
\hline VOT 1 & 0.9764 & 0.6821 & 0.7948 & 0.9762 & 0.6643 \\
VOT 2 & 0.9737 & 0.7518 & 0.7878 & 0.9734 & 0.5341 \\
VOT 3 & 0.9822 & 0.6938 & 0.8073 & 0.9823 & 0.6572 \\
VOT 4 & 0.9672 & 0.7736 & 0.9245 & 0.9677 & 0.5758 \\
VOT 5 & 0.9781 & 0.7894 & 0.9164 & 0.9867 & 0.5821 \\
VOT 6 & 0.9925 & 0.7997 & 0.8977 & 0.9995 & 0.5692 \\
VOT 7 & 0.9222 & 0.7992 & 0.8323 & 0.9898 & 0.5858 \\
VOT 8 & 0.9874 & 0.6819 & 0.9770 & 0.9924 & 0.5862 \\
VOT 9 & 0.9636 & 0.7735 & 0.9342 & 0.9854 & 0.5910 \\
\hline
\end{tabular}

Table No. 6: A Summary of ANOVA for response $Y_{1}$ for fitting to quadratic model for verapamil hydrochloride Organogel

\begin{tabular}{lllllll}
\hline Source & Sum of Squares & df & Mean Square & F-value & p-value & \\
\hline Model & 164.98 & 5 & 33 & 18.33 & 0.0186 & significant \\
A-Soyalecithin & 54.9 & 1 & 54.9 & 30.49 & 0.0117 & \\
B-Cholesterol & 44.12 & 1 & 44.12 & 24.5 & 0.0158 & \\
AB & 40.96 & 1 & 40.96 & 22.75 & 0.0175 & \\
$A^{2}$ & 1.26 & 1 & 1.26 & 0.7021 & 0.4636 & \\
$B^{2}$ & 23.74 & 1 & 23.74 & 13.18 & 0.036 & \\
Residual & 5.4 & 3 & 1.8 & & & \\
Cor Total & 170.38 & 8 & & & & \\
Std. Dev. & 1.34 & & $\mathrm{R}^{2}$ & & & \\
Mean & 61.41 & & Adjusted $\mathrm{R}^{2}$ & 0.9683 & & \\
C.V. $\%$ & 2.18 & & Predicted $\mathrm{R}^{2}$ & 06831 & & \\
\hline
\end{tabular}


Table no. 7: Simultaneous optimal solution by RSM for Verapamil hydrochloride Organogel

\begin{tabular}{llllllllll}
\hline Response & $\begin{array}{l}\text { Pred. } \\
\text { Mean }\end{array}$ & $\begin{array}{l}\text { Pred. } \\
\text { Median }\end{array}$ & Observed & $\begin{array}{l}\text { Std. } \\
\text { Deviation }\end{array}$ & $\begin{array}{l}\text { SE } \\
\text { Mean }\end{array}$ & $\begin{array}{l}\text { 95\% CL } \\
\text { Low }\end{array}$ & $\begin{array}{l}\text { 95\% CL } \\
\text { High }\end{array}$ & $\begin{array}{l}\text { 95\%TL } \\
\text { Low }\end{array}$ & $\begin{array}{l}\text { 95\% } \\
\text { High }\end{array}$ \\
\hline $\begin{array}{l}\text { Drug } \\
\text { Release }\end{array}$ & 96.87 & 96.87 & 97.92 & 2.06 & 1.53 & 91.97 & 101.77 & 77.73 & 116.01 \\
\hline
\end{tabular}

Table no. 8: Stability study data of optimized formulation of Verapamil hydrochloride Organogel

\begin{tabular}{|c|c|c|c|c|c|}
\hline \multirow{2}{*}{ Stability conditions } & \multirow{2}{*}{ Months } & \multicolumn{4}{|c|}{ Evaluation Parameters } \\
\hline & & Phase separation & $\mathrm{pH}$ & Spreadability & Drug Content (\%) \\
\hline \multirow{4}{*}{$2-8 \div C$} & 0 & No Change & $6.20 \pm 0.03$ & $31.5 \pm 0.01$ & $98.68 \pm 0.014$ \\
\hline & 1 & No Change & $6.32 \pm 0.04$ & $30.8 \pm 0.04$ & $99.11 \pm 0.020$ \\
\hline & 2 & No Change & $6.11 \pm 0.02$ & $30.5 \pm 0.02$ & $98.77 \pm 0.017$ \\
\hline & 3 & No Change & $6.58 \pm 0.02$ & $30.1 \pm 0.04$ & $99.16 \pm 0.023$ \\
\hline \multirow{4}{*}{ Room Temperature } & 0 & No Change & $6.70 \pm 0.03$ & $31.2 \pm 0.01$ & $97.68 \pm 0.032$ \\
\hline & 1 & No Change & $6.59 \pm 0.04$ & $30.7 \pm 0.04$ & $98.25 \pm 0.019$ \\
\hline & 2 & No Change & $6.24 \pm 0.02$ & $30.3 \pm 0.02$ & $97.45 \pm 0.013$ \\
\hline & 3 & No Change & $6.38 \pm 0.02$ & $30.1 \pm 0.04$ & $97.56 \pm 0.025$ \\
\hline \multirow{4}{*}{$40 \pm 2 \circ \mathrm{C} / 75 \% \mathrm{RH} \pm 5 \%$} & 0 & No Change & $6.35 \pm 0.03$ & $31.5 \pm 0.01$ & $99.12 \pm 0.014$ \\
\hline & 1 & No Change & $6.44 \pm 0.04$ & $31.0 \pm 0.04$ & $98.03 \pm 0.020$ \\
\hline & 2 & No Change & $6.55 \pm 0.02$ & $30.8 \pm 0.02$ & $97.41 \pm 0.023$ \\
\hline & 3 & No Change & $6.31 \pm 0.02$ & $30.4 \pm 0.04$ & $97.75 \pm 0.013$ \\
\hline
\end{tabular}

Zero order plot for VH organogl

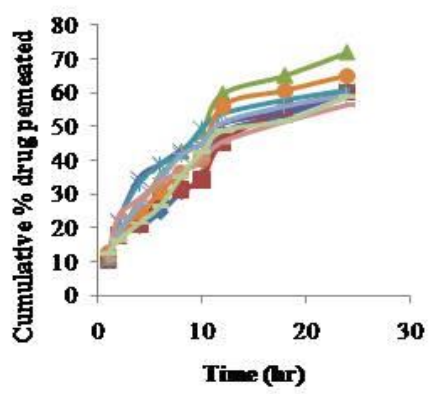

- TV1
- TV2
- TV3
- TV4
- TV5
- TV6
- TV7
- TV8
- TV9

Higuchi plat for VH organeged

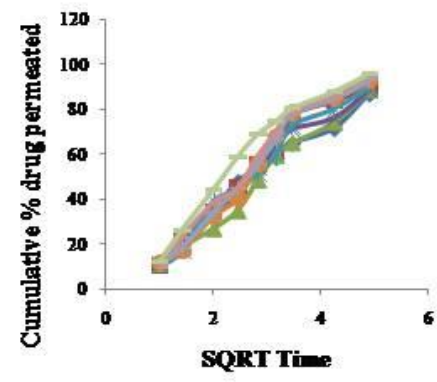

korseneyer-Peppad plot for VH organogel

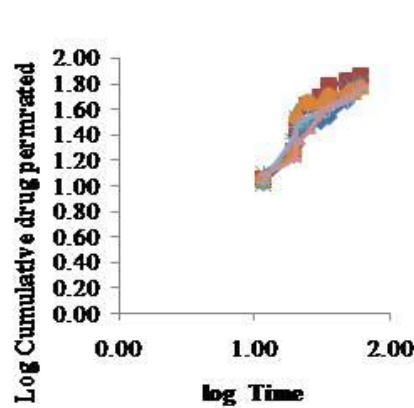

$\leftarrow$ TV2
- TV3
- TV4
- TV5
$*$ TV6
- TV7
+ TV8
- TV9

First order plot for VH organogel

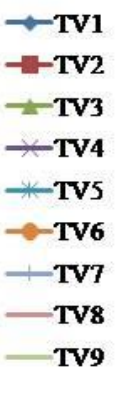

Fig. No. 1: Kinetics of drug release for formulations of verapamil hydrochloride organogel 
Factor Coding: Actual

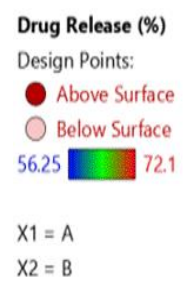

Factor Coding: Actual

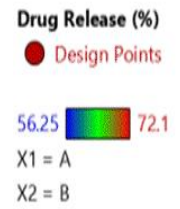

3D Surface
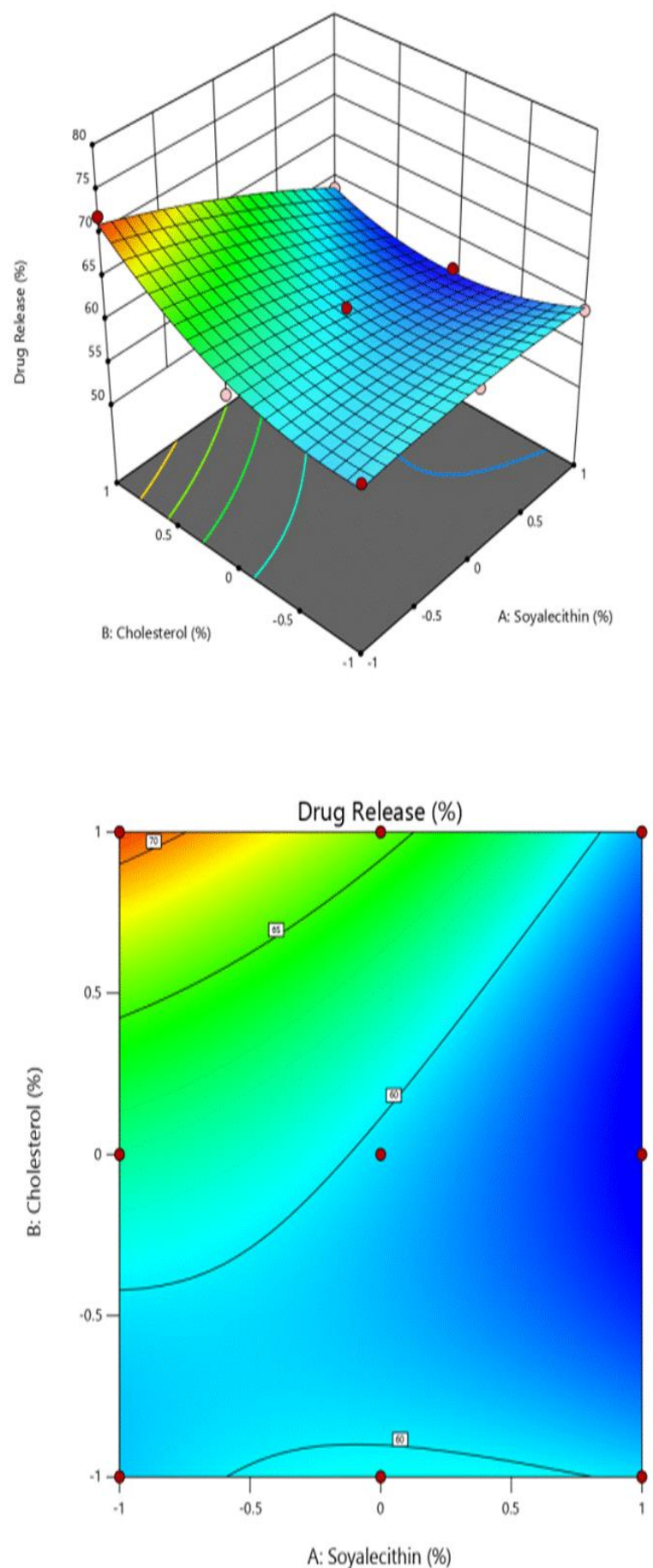

Fig. No. 2: Response Surface plot and Counter Plot for verapamil hydrochloride organogel

\section{CONCLUSION:}

Pluronic organogel was thought to penetrate into skin, interact and disorganize the lipid to penetrate the lipid layers of stratum corneum. However, improved topical drug delivery has been attributed to the biphasic drug solubility, the desired content and modifications of skin barrier function by the organogel components. The finding of the present study suggest that the prepared verapamil hydrochloride organogel containing Pluronic Lecithin were observed to be safe, stable drug delivery system and hold promise for improved bioavailability and better management of hypertension on long term basis. The optimized formulation of organogel 
should be carried out on human volunteers to confirm improved pharmacokinetic parameters.

\section{ACKNOWLEDGEMENT:}

The authors would like to acknowledge Yarrow chemicals Pvt. Ltd., Mumbai (India), for providing sample of verapamil hydrochloride. The authors are very thankful to the Management of Vidyabharati College of Pharmacy, Amravati, for providing necessary facilities and encouragement to carry out the work.

\section{Conflict of Interest: Nil}

\section{REFERENCES:}

1. Shams MS, Alam MI, Ali A, Sultana Y, Aquil M., Pharmacodynamics of a losartan transdermal system for the treatment of hypertension. DDIP, 36(4), 2010, 385-392.Mantry S, 2. Patnaik A, Sriram N, Bharath Raju V. Formulation of bifonazole organogel as a novel topical drug delivery system. IJP; 2013; 3(1): 1-8.

2. Shoo C, Styanarayan K, Bomma NG et.al. Formulation and evaluation of bifonazole organogel for the application of topical drug delivery system. Der pharmacia sinica; 2013; 4(3):67-74.

3. Surber C, Davis FA. Bioavailability and bioequivalance. In: Walter KA (Ed.) Dermatological and transdermal formulations, Marcel Dekker Inc. New York; 2002; 119: 401.

4. Schipunov YA, Dueerrschmidt T, Hoffmann $H$. Electroheological effects in lecithin organogels with water and glycerol. J. Colloid Interface Science; 1999; 212:390-401.

5. Shams MS, Alam MI, Ali A, Sultana $Y$, Aquil M., Pharmacodynamics of a losartan transdermal system for the treatment of hypertension. DDIP, 36(4), 2010, 385-392.

6. Pandey MS, Belgamwar VS, Gattani S, Surana SJ, Tekade A. Pluronic lecithin organogel as a topical drug delivery system. Drug Delivery; 2010; 17: 38-47.

7. Pandey MS, Belgamwar VS, Surana SJ. Topical delivery of flurbiprofrn from pluronic lecithin organogel. IJPS; 2009: 87-90.

8. Murapur SC, Wali DS, Hunasagi BS, Chetankumar M, Patil RG., Formulation and evaluation of transdermal drug delivery system of verapamil hydrochloride., JDDT, 2018, 8(6), 70-77.

9. Dawange SR, Khadbadi SS, Saboo SS., Formulation and evaluation of floating tablets of verapamil hydrochloride by using gastroretentive technology., Int. J. Pharm. Sci. Rev. Res. 2015, 34(1), 263.269.

10. Patel R, Sindhu A, Bharath S, Madhavan V, Sorbitan monostearate based organogels for topical delivery of clotrimazole. IJPCS, 2013; 2(3), 1246-1252.

11. Choukse R, Sangameswaran B. Formulation and evaluation of pluronic lecithin organogel of flurbiprofen. JBPR. 2012; 1(1): 1-7.

12. Agrawal V, Gupta V, Ramteke S, Trivedi P. Preparation and evaluation of tubuler micelles of pluronic lecithin organogel for transdermal delivery of sumitriptan. AAPS PharmSciTech. 2010, 2(4): 1718-1725.

13. Murdan $S, A$ review of pluronic lecithin organogel as a topical and transdermal drug delivery system. Hospital Pharmacist. 2005; 12: 267-279.

14. Jadhav KR, Kadam VJ, Pisal SS. Formulation and evaluation of lecithin organogel for topical delivery of fluconazole. Current drug delivery. 2009; 6: 174-183.

15. Nasseri AA, Aboofazeli R, Zia H, Needham TE. Lecithin stabilized microemulsion: an organogel for topical application of ketorolac trimethamine I: Phase behaviour studies. IRan J. Pharm Res. 2003; 2: 59-61.

16. Mutimer MN, Riffkin C, Hill JA, Murray E, Glickman $\mathrm{ME}$, Cyr CN. Modern ointment base technology II comparative evaluation of bases. J.Am.Pharm. Assoc, 1956; 45: 212-18.

17. Willaimann $\mathrm{H}$, Walde $\mathrm{P}$, Luisil $\mathrm{PL}$, Gazzaniga $\mathrm{A}$, Stroppolo F. Lecithin organogels as matrix for transdermal transport of drugs. J.Pharm.Sci., 1992; 81; (9): 871-874.

18. Bhatia A, Singh, Bhupinder, Raza Kaisar, Wadhwa S, Katare OP. Tamoxifen loaded lecithin organogel (LO) for topical application: Development, optimization and characterization. IJP. 2013; 444: 47-59.

19. Atram SC, Joshi AV, Bobade NN, Wankhade VP, Pande $\mathrm{SD}$., Formulation and evaluation of pluronic lecithin clotrimazole organogel for the topical delivery, IAJPR, 2019, 9(01), 1860-1864.

20. Atram SC, Pande SD., A statistical approach to the development of fast disintegrating tablets of telmisartan using co-processed superdisintegrant, IJPSR, 2019, 10(11), 1000-1010. 